\title{
Parameterization of BFO Algorithm for the Improved Functionality of MFKM Technique for Better Pathological Identification in Brain MR Image
}

\author{
Anitha Narayanan, Yudong Zhang, Pallikonda Rajasekaran Murugan, Vishnuvarthanan \\ Govindaraj, Vigneshwaran Senthilvel, Sakthivel Sankaran
}

\begin{abstract}
Intensity inhomogeneity, high level of noise, partial volume effect and poor image contrast are the major artefacts in medical image segmentation. Any of these artefacts might lead to unclear boundaries of tissues, hence the segmentation of tissues in the MR brain image cannot be determined with high accuracy, and this would be a problem to the radiologists to diagnose or to start the treatment because of the lack of facility to operate over the brain in in-vivo condition. This makes the radiologist and surgeons/experts to take time to come for the conclusion on pathology of a particular patient. So, the radiologists and experts need to give more exertion when this condition is applied for many patients at a day, to diagnose and to start treatment. To make this effortless to them, also for accurate diagnosis, this research paper provides an robust algorithm using the Modified Fuzzy K-Means (MFKM) and Bacteria Foraging Optimization (BFO) algorithm, which segments the abnormal tissues among the normal tissues from MR brain images with high accuracy. The accuracy of the Improved MFKM (IMFKM) algorithm is obtained in terms of Sensitivity and Specificity, and the proposed algorithm proves better segmentation results than the other conventional algorithms.
\end{abstract}

Keywords: Bacteria Foraging Optimization, Magnetic Resonance (MR) Image Segmentation, Modified Fuzzy K Means, Tissue Segmentation, Tumor Identification.

\section{INTRODUCTION}

From the world population of 7.9 billion, approximately 18.1 million people are affected by cancer right now. It

Revised Manuscript Received on December 16, 2019.

* Correspondence Author

Anitha Narayanan*, department of ECE, Kalasalingam Academy of Research and Education (Kalasalingam University), Srivilliputtur, India. Email: anithaa06@gmail.com

Yudong Zhang, Professor, F26 Informatics Building, Department of Informatics, University of Leicester, University Road, Leicester, LE1 7RH, UK. yudongzhang@ieee.org

Pallikonda Rajasekaran Murugan, Department of ECE, Kalasalingam Academy of Research and Education (Kalasalingam University) Srivilliputtur, India. Email: m.p.raja @klu.ac.in

Vishnuvarthanan Govindaraj, Department of BME, Kalasalingam Academy of Research and Education (Kalasalingam University) Srivilliputtur, India. Email: gvvarthanan@gmail.com

Vigneshwaran Senthilvel, Department of ECE, Kalasalingam Academy of Research and Education (Kalasalingam University) Srivilliputtur, India. Email: a.s.vigneshwaran@klu.ac.in

Sakthivel Sankaran, Department of BME, Kalasalingam Academy of Research and Education (Kalasalingam University) Srivilliputtur, India. Email: sakthivelsankaran92@gmail.com happened due to the lack of lifestyle among the people, which results in the increasing of incidence rate of the cancer population every year around the world. The awareness of cancer symptoms is binged more in this decade in order to reduce the mortality rate. Speedy and accurate diagnosing of the patients who suffer from cancer is a major leap in this idea to cope up with the cancer population, also it results in accurate conclusion of pathologies in the patients, leading to start with the treatment as soon as possible, hence the lifespan of the patient will be extended to achieve/reach minimum 5 -years prevalence. In this research paper, the authors have introduced an effective objective function by changing the value of centroid/cluster center in the standard Fuzzy K-Means (FKM) algorithm in order to effectively segment the abnormal brain tissues. This have been elaborated in the methodology section IV. Also, the technique proposed tries to pact the poor image contrast, intensity inhomogeneity and image noise in an unsurpassed way, which have been showed in the results section $\mathrm{V}$.

\section{LITERATURE SURVEY}

The usage of term 'K-Means' was first publicized by the research work contributed by James MacQueen in 1967 [1]. But, in the year of 1957, Stuart Lloyd promulgated the standard K-Means algorithm as a measure to be applied for pulse-code modulation in Bell Telephone Laboratories, until it was published in the year 1982; the method was quite unfamiliar to many [2]. In 1965, Forgy [3] published the same essential method, which is why it is so called as Lloyd-Forgy. In 1965, the term 'Fuzzy' was introduced by Zadeh [4]. It was an extended version of the classical set theory, and it tends to be a powerful tool for soft partitioning of data. The ideology related to the use of fuzzy set theory [1] for clustering was first connoted by Ruspini $[5,6]$. The author has suggested the fuzzy clustering because it is one of the unsupervised algorithms that reduce the rate of human intervention, and the least requirement of prior information of the data to be clustered. Thus, it is used in many applications including medical image analysis, and the author has recommended it especially for brain image analysis as it is capable of handling the extremities such as complex structured unsupervised data with wider range of uncertainty. 
The modification of FKM is done because it converges quickly to cluster the pixels, and they have the general property to lose vital pixels while undergoing clustering, which mostly ends in under segmentation. The weighted value function is involved to cluster maximum number of pixels available in the input data during the clustering process. Hence, under segmentation gets diminished at a higher rate. Color conversion-based segmentation merged with $\mathrm{K}$-means render the tracking of tumor object in brain MRI by converting grayscale image into color space image, and the segmentation operation of the image is controlled by labelling using cluster index which helps to detect the exact tumor size and topology [7]. The algorithm functions quicker, which makes it recommendable for clinical/real-time applications. [8] have introduced an unsupervised segmentation of MR brain images using Dual Tree Complex Wavelet Transform (DT-CWT) and K-means algorithm. The augmentation of TC value is required by the algorithm. Also, the algorithm segments T1-W MR brain images only, which needs to be expanded. MiMSeg algorithm was developed by [9] for the automatic identification of GBM tumors in DWI brain image, and it is the mixture of $\mathrm{K}$-means clustering and Gaussian Mixture Model (GMM). The application of the algorithm have utilized only on one type of tumor and it could be extended to identify other types of tumors present in different MR brain image sequences.

\section{DESCRIPTION OF DATASET}

The dataset has totally $115 \mathrm{MR}$ brain images, includes 17 clinical images, 21 images from Harvard BrainWeb Repository, 50 images from BRATS-2013 Challenge Dataset [10] and 27 images from BrainWeb: Simulated Brain Database [11]. The clinical images acquired using MRI scanner (developed by Siemens-Aera) with magnetic field intensity of 1.5 Tesla. The proposed algorithm was tested on different MR sequences of brain images, such as the T1-Weighted (T1-W), T2-Weighted (T2-W), Fluid Attenuated Inversion Recovery (FLAIR), Multi Planar Reconstruction (MPR) and Contrast Enhancement (CE), also with three different axes namely the axial, sagittal and coronal. The dataset consists of different types of tumors, which are, Glioma, Astrocytoma, Meningioma, Metastatic Carcinoma, Pituitary Adenomas and Primitive Neuro Ectodermal.

\section{METHODLOGY}

Fig. 1. represents the block diagram of the proposed method. The input MR brain images are pre-processed by Contrast limited Adaptive Histogram Equalization (CLAHE), which has the advantage over the Adaptive Histogram Equalization (AHE), and CLAHE has the ability to avoid the noise amplification developing in the homogeneous regions. Thus, the IMFKM deals the poor image contrast setback, which is one of the major problems in MR brain image segmentation. The pre-processed image is given as input to MFKM algorithm for the segmentation process, and the segmented results is further optimized using BFO (introduced by passino [12]) algorithm. The optimum threshold by BFO is utilized to get optimum segmentation results.

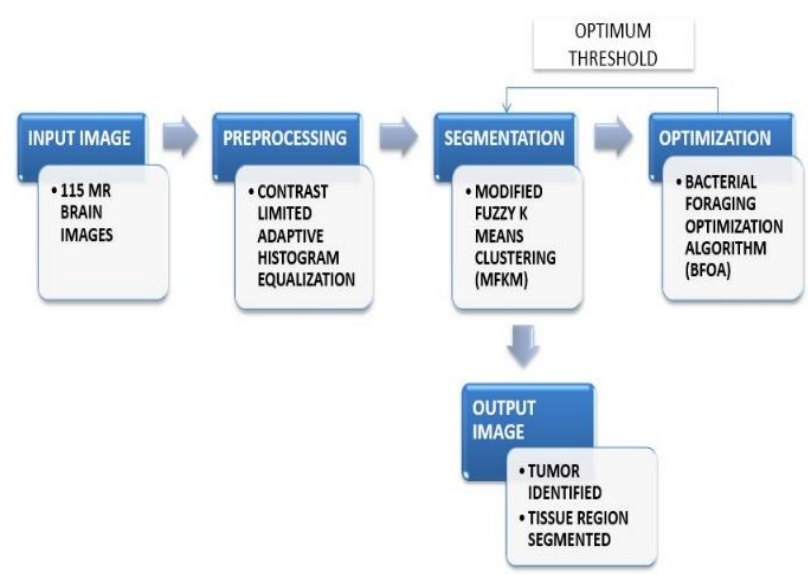

Fig. 1. Block diagram of the proposed IMFKM algorithm.

\section{A. The Step by Step Procedure of proposed IMFKM Algorithm}

- Initialising the cluster based on the number of peaks from CLAHE.

- Membership function $\left(u_{i j}\right)$ assigned to each data point $\left(x_{i}\right)$ corresponding to each cluster and the centroid $\left(C_{j}\right)$ is calculated. (1) and (2) represents the membership function and centroid, correspondingly.

$$
u_{i j}=\left[\left(d_{i j}\right)^{\frac{1}{m-1}} \sum_{l=1}^{K}\left(\frac{1}{d_{i j}}\right)^{\frac{1}{m-1}}\right]^{-1}
$$

Where, ' $K$ ' defines the number of clusters, $l=1$ illustrates the first cluster and ' $m$ ' represents the fuzziness co-efficient.

$$
C_{j}=\frac{\sum_{i=1}^{N} u_{i j}^{* m} x_{i}}{\sum_{i=1}^{N} u_{i \bar{j}}^{* m}}
$$

Where, $u_{i j}^{*}$ represents the updated membership function.

- The clustering process is done by Euclidean distance $\left(d_{i j}\right)$ with the help of $\left(C_{j}\right)$ and Weighted value $\left(W_{i j}\right)$.

- After each iteration, $u_{i j}$ and $C_{j}$ are updated in using (3). The objective function of segmentation is given in (3) and it is minimized to find the best location for the clusters.

$$
\begin{gathered}
J_{k}=\sum_{j=1}^{K} \sum_{i=1}^{N} u_{i j}^{* m} d_{i j} \\
W_{i j}=\frac{\sum_{x_{n} \in N_{i}^{j}} \frac{1}{1+\alpha\left(d_{i j}\right)^{2}}}{\sum_{x_{n} \in N_{i 11+\alpha\left(d_{i j}\right)^{2}}}}
\end{gathered}
$$

- In (3), $J_{k}$ ' states the segmented output image, ' $k$ ' defines the total number of iterations, ' $i$ ' and ' $j$ ' are the rows and columns of the input image, respectively. ' $N$ ' indicates the total number of pixels present in the input image 
International Journal of Innovative Technology and Exploring Engineering (IJITEE) ISSN: 2278-3075, Volume-9, Issue-2S2, December 2019

\begin{tabular}{|c|c|c|c|c|c|c|}
\hline & $\begin{array}{l}\text { Type of } \\
\text { Tumor }\end{array}$ & $\begin{array}{c}\text { MR } \\
\text { Sequence }\end{array}$ & $\begin{array}{c}\text { Input } \\
\text { Images }\end{array}$ & CLAHE Results & MFKM Results & IMFKM Results \\
\hline A. & $\begin{array}{l}\text { Low-grade } \\
\text { Glioma }\end{array}$ & $\begin{array}{c}\text { T1-W } \\
\text { Axial } \\
\text { with CE }\end{array}$ & & & & 8 \\
\hline B. & $\begin{array}{l}\text { high-grade } \\
\text { Glioma }\end{array}$ & $\begin{array}{c}\mathrm{T} 1-\mathrm{W} \\
\text { Axial } \\
\text { with CE }\end{array}$ & & & & \\
\hline C. & $\begin{array}{l}\text { High-grade } \\
\text { Astrocytoma }\end{array}$ & $\begin{array}{l}\text { T2-W } \\
\text { Axial }\end{array}$ & & & & \\
\hline D. & Meningioma & $\begin{array}{l}\text { T2-W } \\
\text { Sagittal } \\
\text { with CE }\end{array}$ & & & & \\
\hline E. & $\begin{array}{l}\text { Metastatic } \\
\text { Bronchogeni } \\
\text { c Carcinoma }\end{array}$ & $\begin{array}{c}\text { T2-W } \\
\text { Axial } \\
\text { with CE }\end{array}$ & & & & \\
\hline F. & $\begin{array}{c}\text { Pituitary } \\
\text { Adenomas }\end{array}$ & $\begin{array}{c}\text { T1-W } \\
\text { Axial } \\
\text { with CE }\end{array}$ & & & & \\
\hline G. & PNET & $\begin{array}{l}\mathrm{T} 1-\mathrm{W} \\
\text { Coronal }\end{array}$ & & & & \\
\hline
\end{tabular}

Fig. 2. Segmented results of clinical and Harvard BrainWeb dataset.

\begin{tabular}{|c|c|c|c|c|c|}
\hline $\begin{array}{c}\text { MR } \\
\text { Sequence } \\
\text { s }\end{array}$ & $\begin{array}{l}\text { Noise } \\
\text { levels }\end{array}$ & $\begin{array}{c}\text { Input } \\
\text { Images }\end{array}$ & $\begin{array}{c}\text { CLAHE } \\
\text { Results }\end{array}$ & $\begin{array}{l}\text { MFKM } \\
\text { Results }\end{array}$ & $\begin{array}{c}\text { IMFKM } \\
\text { Results }\end{array}$ \\
\hline $\begin{array}{c}\text { PD } \\
\text { Axial }\end{array}$ & $1 \%$ & & & & \\
\hline $\begin{array}{c}\mathrm{T} 1 \\
\text { Coronal }\end{array}$ & $9 \%$ & & & & \\
\hline $\begin{array}{c}\mathrm{T} 2 \\
\text { Sagittal }\end{array}$ & $1 \%$ & & & & \\
\hline
\end{tabular}

Fig. 3. Segmented results of BrainWeb: Simulated Brain Database.

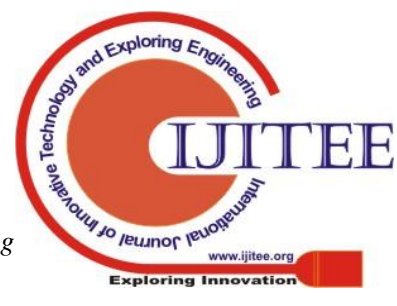


Parametrization of BFO Algorithm for the Improved Functionality of MFKM Technique for Better Pathological Identification in Brain MR Image

\begin{tabular}{cccccc}
\hline $\begin{array}{c}\text { MR } \\
\text { Sequences }\end{array}$ & $\begin{array}{c}\text { Type of } \\
\text { tumor }\end{array}$ & $\begin{array}{c}\text { Input } \\
\text { Images }\end{array}$ & $\begin{array}{c}\text { CLAHE } \\
\text { Results }\end{array}$ & $\begin{array}{c}\text { MFKM } \\
\text { Results }\end{array}$ & $\begin{array}{c}\text { IMFKM } \\
\text { Results }\end{array}$ \\
\hline \multirow{2}{*}{ FLAIR } & $\begin{array}{l}\text { Low- } \\
\text { grade }\end{array}$ & & & & \\
& T1-CE & $\begin{array}{l}\text { High- } \\
\text { grade }\end{array}$ & & &
\end{tabular}

Fig. 4. Segmented results of BRATS-2013 Challenge dataset.

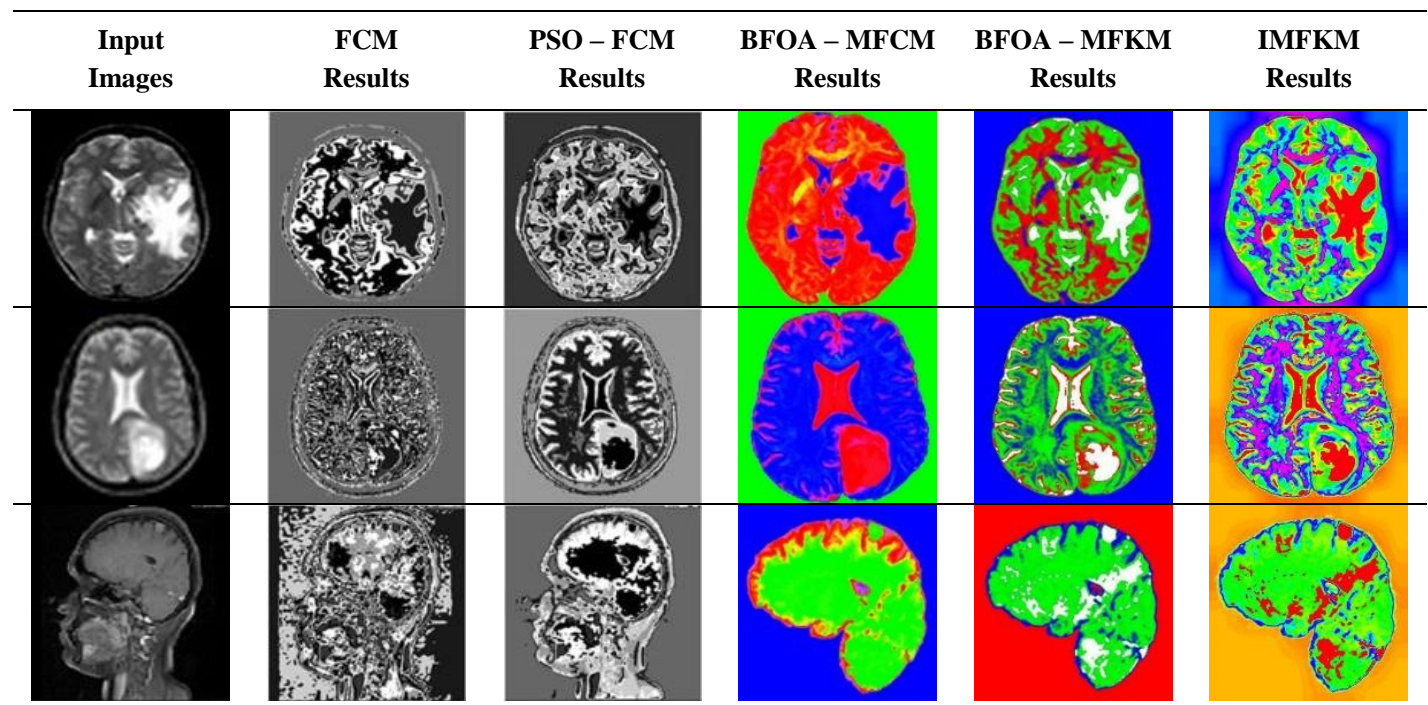

Fig. 5. Comparison of segmented results of proposed method with other soft computing techniques.

Table- I: Performance Analysis Using Comparison Metrics

\begin{tabular}{|c|c|c|c|c|c|}
\hline \multirow{2}{*}{ Comparison Metrics } & \multicolumn{5}{|c|}{ Algorithms } \\
\cline { 2 - 6 } & FCM & $\begin{array}{c}\text { PSO-FC } \\
\mathbf{M}\end{array}$ & $\begin{array}{c}\text { BFO-MFC } \\
\mathbf{M}\end{array}$ & $\begin{array}{c}\text { BFO-MFK } \\
\mathbf{M}\end{array}$ & \multirow{2}{*}{ IMFKM } \\
\hline MSE & 0.1425 & 0.1121 & 0.1015 & 0.2166 & 0.1092 \\
\hline PSNR in dB & 56.51 & 57.63 & 58.064 & 54.855 & 57.749 \\
\hline TC in \% & 23.559 & 22.544 & 25.231 & 23.354 & 32.76 \\
\hline DOI in \% & 38.134 & 36.79 & 40.29 & 37.865 & 49.35 \\
\hline Computational Time in Seconds & 24.775 & 22.365 & 7.928 & 10.360 & 8.098 \\
\hline Memory Requirement in Bytes & $\begin{array}{c}4320000 \\
0\end{array}$ & 155000000 & 1687 & 1810 & 1739 \\
\hline
\end{tabular}

and ' $K$ ' represents the number of clusters into which the given input image is to be partitioned. The weighted value function is given in (4), $W_{i j}$ ( $i^{\text {th }}$ pixel belonging to the $j^{\text {th }}$ cluster) is used to update the $u_{i j}$ value of every pixel and $C_{j}$ are updated. In addition to the membership value of the pixel, IMFKM is effectively lessen the effect of noise acting upon a pixel [13] using the intensity levels of the neighbouring pixels. Moreover, the presence of $W_{i j}$ in the proposed IMFKM algorithm helps in minimizing the convergence rate of the algorithm $[14,15]$ and it guarantees that every pixel in the input MR brain images are grouped by IMFKM.

- The re-evaluation of IMFKM segmented result is done using the optimum threshold which is obtained by the objective function of BFO algorithm as represented in (5).

$$
J(i, j, k, l)=J(i, j, k, l)+J_{c c}\left(\theta^{i}(j, k, l), P(j, k, l)\right)
$$

Published By:

Where,
$J_{c c}\left(\theta^{i}(j, k, l), P(j, k, l)\right)=$ Swarm attractant cost function. $\theta^{i}(j, k, l)=$ The location of $i^{\text {th }}$ bacterium at $j^{\text {th }}$ chemotactic, $k^{\text {th }}$ reproductive and $l^{\mathrm{h}}$ elimination and dispersal step.

Number of parameters to be optimized

which are present in each bacterium.

- The final segmentation upon $J$, arbitrated from MFKM is obtained using BFO threshold function.

- Finally, tissue segmentation and tumor identification take place concurrently.

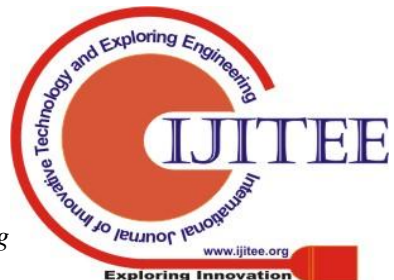




\section{RESULT AND DISCUSSION}

Figures Fig. 2 to Fig. 4 elucidate the segmentation results of the proposed algorithm for the dataset mentioned in section III. In Fig. 2. the complicated cases have been taken to prove the efficiency of the proposed IMFKM algorithm, which are very difficult to diagnose during clinical procedures. From Fig. 2. (A) to $(G)$, IMFKM results empower the MFKM results, and in that, the tissues are clearly segmented. Moreover, Fig. 2. (A), (D), (E) and (G) prove the excellence of the proposed algorithm, which the tumor regions are identified with high level of accuracy. Fig. 3. represents the results of BrainWeb: Simulated Brain Database for different noise levels, where IMFKM give best results than MFKM results, which shows the proposed algorithm works well even on the noisy images. Similarly, Fig. 4. represents the outstanding segmenttion results of IMFKM than the MFKM for the BRATS-2013 challenge dataset, which explicitly state that the IMFKM produces results better than the MFKM segmentation results.

Fig. 5. gives the assessment of proposed IMFKM algorithm with the other soft computing methods such as conventional Fuzzy C-Means (FCM), Particle Swarm Optimization based FCM (PSO-FCM) [16], BFO based Modified Fuzzy C-Means (BFO-MFCM) [17] and BFO based Modified Fuzzy K-Means (BFO-MFKM) [18]. Table I and II give the comparison of benchmark metrics such as Mean Squared Error (MSE), Peak Signal to Noise Ratio (PSNR), Jaccard Tanimoto Cofficient (TC), Dice Overlap Index (DOI), sensitivity, specificity, computational time (C.Time) and memory requirement.

Table- II: Performance Analysis Using Sensitivity and Specificity Values

\begin{tabular}{|c|c|c|}
\hline Algorithms & Sensitivity & Specificity \\
\hline FCM & 0.7992 & 0.7126 \\
\hline PSO-FCM & 0.8701 & 0.7965 \\
\hline BFOA-MFKM & 0.9032 & 0.9821 \\
\hline BFOA-MFCM & 0.9048 & 0.9825 \\
\hline IMFKM & 0.9356 & 0.9921 \\
\hline
\end{tabular}

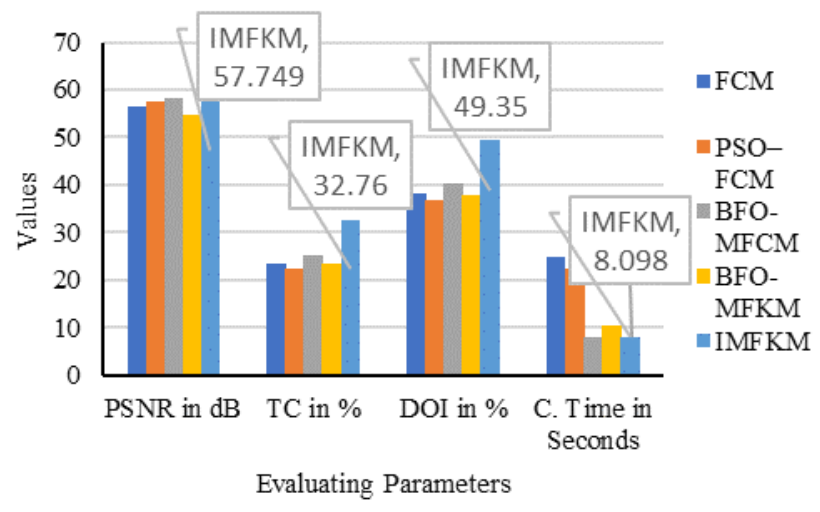

Fig. 6. Comparison of PSNR, TC, DOI and Computational Time

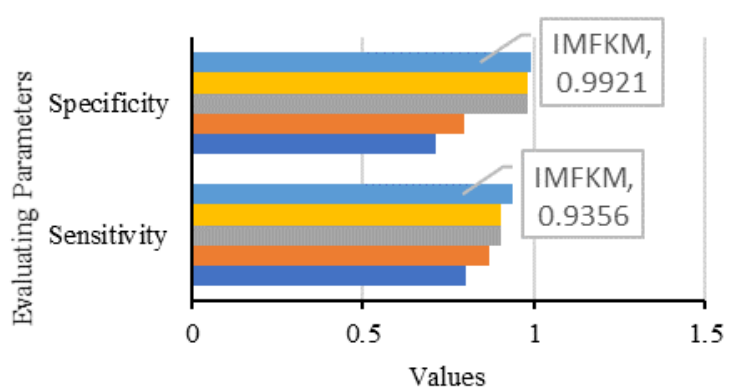

$\begin{array}{ll}=\mathrm{IMFKM} & \square \mathrm{BFOA}-\mathrm{MFCM} \\ =\mathrm{PSO}-\mathrm{FCM} & \square \mathrm{FCM}\end{array}$

Fig. 7. Comparison of Sensitivity and Specificity.

The graphical representations for the comparison of results of Table I and Table II have shown in Fig. 6 and Fig. 7 respectively, to have clear understanding of the proposed algorithm, IMFKM.

\section{CONCLUSION}

The proposed IMFKM algorithm deals 115 MR brain images with different type of tumors and complicated cases, also for different types of MR sequences and for various noise levels. The IMFKM also dealt with intensity inhomogeneity, noise and poor image contrast, which prove the over-all efficacy of IMFKM with other algorithms as mentioned in results section V. Further decrease in MSE and PSNR values, and with subsequent increase of TC and DOI values are considered to be the furture scope of this research work.

\section{ACKNOWLEDGMENT}

The authors thank the Department of Electronics and Communication Engineering of Kalasalingam Academy of Research and Education (Kalasalingam University), Tamilnadu, India for permitting to use the computational facilities available in open source research laboratory. Also, the authors thank Dr. K. G. Srinivasan, MDRD, Consultant Radiologist and Dr. K. P. Usha Nandhini, DNB, KGS Advanced MR \& CT Scan - Madurai, Tamilnadu, India, for supporting the research with the patient information.

\section{REFERENCES}

1. J. MacQueen, "Some methods for classification and analysis of multivariate observations," Proc. of the $5^{\text {th }}$ Berkeley Symposium on Mathematical Statistics and Probability, University of California Press, vol. 1, 1967, pp. 281-297.

2. S. Lloyd, "Least squares quantization in PCM," IEEE Trans. Inform. Theory, vol. 28 (2), March 1982, pp. 129-137. doi: 10.1109/TIT.1982.1056489

3. E. W. Forgy, "Cluster analysis of multivariate data: efficiency versus interpretability of classifications," Biometrics, vol. 21 (3), 1965, pp. 768-769.

4. L. A. Zadeh, "Fuzzy Sets," Inf. Control, vol. 8 (3), 1965, pp. 338-353.

5. E. H. Ruspini, "A new approach to clustering," Inf. Control, vol. 15, 1969, pp. 22-32.

6. E. H. Ruspini, "Numerical methods for fuzzy clustering," Inf. Sci., vol 2 (3), 1970, pp. 319-350.

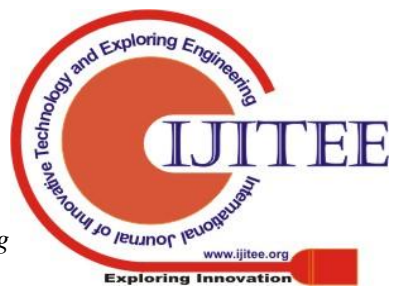


7. L. Juang and M. Wu, "MRI brain lesion image detection based on color converted k-means clustering segmentation," Meas., vol. 43 (7), 2010, pp. 941-949.

8. J. Zhang, W. Jiang, R. Wang and L. Wang, "Brain MR image segmentation with spatial constrained $\mathrm{k}$-mean algorithm and dual-tree complex wavelet transform," J. Med. Syst., vol. 38, 2014, pp. 1-6.

9. F. Binczyk, B. Stjelties, C. Weber, M. Goetz, K. Meier-Hein, H. Meinzer, B. Bobek-Billewicz, R. Tarnawski and J. Polanska, "Mimseg -an algorithm for automated detection of tumor tissue on NMR apparent diffusion coefficient maps," Inf. Sci., vol. 384, 2017, pp. 235-48.

10. Menze et al. "The multimodal brain tumor image segmentation benchmark (BRATS)," IEEE Trans. Med. Imaging, 2015

11. D. L. Collins, A. P. Zijdenbos, V. Kollokian, J. G. Sled, N. J. Kabani, C. J. Holmes and A.C. Evans, "Design and construction of a realistic digital brain phantom," IEEE Trans. Med. Imaging, vol. 17, 1998, pp. 463-468.

12. K. M. Passino, "Biomimicry of bacterial foraging for distributed optimization and control," IEEE Control Syst. Mag., vol. 22, 2002, pp. 52-67.

13. K. Sikka, N. Sinha, P. K. Singh and A. K. Mishra, "A fully automated algorithm under modified fcm framework for improved brain MR image segmentation," Magn. Reson. Imaging, vol. 27 (7), 2009, pp. 994-1004.

14. G. Vishnuvarthanan, M. P. Rajasekaran, P. Subbaraj and V. Anitha, "An unsupervised learning method with a clustering approach for tumor identification and tissue segmentation in magnetic resonance brain images," Appl. Soft Comput., vol. 38, 2016, pp. 190-212.

15. C. T. Chang, J. Z. C. Lai and M. Jeng, "A fuzzy k-means clustering algorithm using cluster center displacement," J. Inf. Sci. Eng., vol. 27 (3), 2011, pp. 995-1009.

16. G. Vishnuvarthanan and M. P. Rajasekaran, "A complete automated algorithm for segmentation of tissues and identification of tumor region in T1, T2, and FLAIR brain images using optimization and clustering techniques," Int. J. Imag. Syst. Tech., vol. 24 (4), 2014, pp. 313-325.

17. V. Anitha, M. P. Rajasekaran, G. Vishnuvathanan, Yudong Zhang and T. Arunprasath, "An automated hybrid approach using clustering and nature inspired optimization technique for improved tumor and tissue segmentation in magnetic resonance brain images," Appl. Soft Comput., vol. 57, 2017, pp. $399-426$.

18. V. Anitha, M. P. Rajasekaran, G. Vishnuvathanan, Yudong Zhang and T. Arunprasath, "Development of a combinational framework to concurrently perform tissue segmentation and tumor identification in T1-W, T2-W, FLAIR and MPR Type magnetic resonance brain images," Expert Syst. Appl., vol. 95, 2018, pp. 280 - 311.

\section{AUTHORS PROFILE}

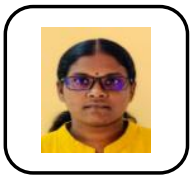

Dr. Anitha. $\mathbf{N}$ has been awarded her $\mathrm{PhD}$ degree from Kalasalingam Academy of Research and Education (Kalasalingam University) in 2019 for her research work in the area of medical image processing. She received her Master's degree in Digital Communication and Networking in 2015 and Bachelor's degree in Electronics and Communication Engineering in 2006. She has three years of work experience as software programmer and two years of teaching experience, also an expertise in students' counselling. She is an active IEEE Member of Pothigai Subsection and IEEE Solid-State Circuits Society Member of IEEE Madras section from March 2018.

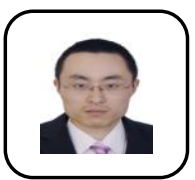

Prof. Yu-Dong Zhang received his $\mathrm{PhD}$ degree from Southeast University in 2010. He worked as a postdoc from 2010 to 2012 in Columbia University, USA, and as an assistant research scientist from 2012 to 2013 at Research Foundation of Mental Hygiene (RFMH), USA. He served as a full professor from 2013 to 2017 in Nanjing Normal University, where he was the director and founder of Advanced Medical Image Processing Group in NJNU. Now he serves as Professor in Department of Informatics, University of Leicester, UK. He was included in "Most Cited Chinese researchers (Computer Science)" by Elsevier from 2014 to 2018 . He was the 2019 recipient of "Highly Cited Researcher" by Web of Science. He won "Emerald Citation of Excellence 2017" and "MDPI Top 10 Most Cited Papers 2015". He was included in "Top Scientist" in Guide2Research. He published over 160 papers, including 16 "ESI Highly Cited Papers", and 2 "ESI Hot Papers". His citation reached 10096 in Google Scholar, and 5362 in Web of Science. He is the fellow of IET (FIET), and the senior members of IEEE and ACM. He is the editor of Scientific Reports, IEEE Transactions on Circuits and Systems for Video Technology, etc. He served as the (leading) guest editor of Information Fusion, Neural Networks, IEEE Transactions on Intelligent Transportation Systems, etc. He has conducted many successful industrial projects and academic grants from NSFC, NIH, Royal Society, and British Council.

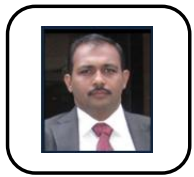

Dr. Pallikonda Rajasekaran Murugan, born in Srivilliputhur, Virudhunagar District of Tamil Nadu in 1980, he had his schooling in the same town and graduated in Electronics and Instrumentation Engineering in 2001 from Shanmugha College of Engineering, Thanjavurand completed his M.Tech. degree in 2002 with second Rank in SASTRA University. He pursued his doctoral programme in Anna University, Chennai. Starting as a Lecturer in 2003, he became Asst. Professor in 2008, Associate Professor in 2009 and Professor in 2012 in Kalasalingam Academy of Research and Education. He had a deep involvement in Bio-signal Processing research. His work on the Image Segmentation for identification of brain tumour and image reconstruction and compression using medical images for diagnosis. Over 150 B.Tech students, 75 M.Tech students, and 8 Doctorates stand testimony for his productivity in Image Processing, Wireless Sensor Networks, and Biomedical Instrumentation research. He has so far published more than 50 papers in national and international journals and conferences. He is a Fellow of Indian Society For Technical Education (ISTE), Institute of Electrical and Electronics Engineers (IEEE), Asia-Pacific Chemical, Biological \& Environmental Engineering Society (APCBEES), Institution of Engineers (India)(IE), International Association of Engineers (IAENG) and International Association of Computer Science and Information Technology (IACSIT)

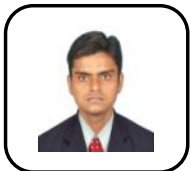

Dr. G. Vishnuvarthanan is an Associate Professor in the Department of Instrumentation Engineering at Kalasalingam University. Dr.Vishnuvarthanan received his Ph.D. in Electronics and Communication Engineering from Kalasalingam University, Tamilnadu in 2015 and completed his Bachelor of Engineering in Instrumentation and Control Engineering with first class in the year 2007 from Arulmigu Kalasalingam College of Engineering, Virudhunagar, Tamilnadu and Masters in VLSI with distinction in the year 2009 from Bharath University, Chennai, Tamilnadu. His areas of interest are Medical image processing and signal processing, and he has significant publications pertaining to these domains.

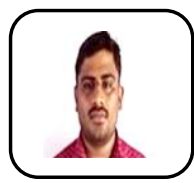

Mr. Vigneshwaran Senthilvel is a full-time research scholar in the Department of Electronics and Communication Engineering at Kalasalingam University. He has completed his M.E in Applied Electronics at Anna University in 2015 and B.E in Electronics and Communication Engineering at Sudharsan Engineering College in 2012, both located at Tamilnadu, India His primary research focus is upon medical image processing.

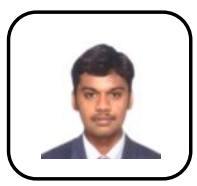

Mr. Sakthivel Sankaran received Bachelor of Engg. in Electronics and Communication Engineering, May 2013 and Master of Engg. in (Biomedical Engineering) at VIT University, Vellore, May 2015. Currently Assistant Professor at Kalasalingam Academy of Research and Education, Krishnankoil. His research interest in Biomechanics, Rehabilitation Engineering, Biomedical Instrumentation. He has so far published more than 09 papers in national and international journals and conferences. He is a Fellow of Indian Society for Technical Education (ISTE), Institute of Electrical and Electronics Engineers (IEEE), Biomedical Engineering Society of India (BMESI). 